\title{
miR-30a-3p suppresses the proliferation and migration of lung adenocarcinoma cells by downregulating CNPY2
}

\author{
HONGTAO WANG $^{1}$, DOMKAM KANMANGNE ${ }^{1}$, RUI LI $^{1}$, ZHONGQING QIAN ${ }^{1}$, \\ XIAOYING XIA ${ }^{1}$, XIAOJING WANG ${ }^{2}$ and TING WANG ${ }^{1}$ \\ ${ }^{1}$ School of Laboratory Medicine, Department of Immunology, Bengbu Medical College; \\ ${ }^{2}$ Anhui Clinical and Preclinical Key Laboratory of Respiratory Disease, Department of Respiration, \\ First Affiliated Hospital, Bengbu Medical College, Bengbu, Anhui 233030, P.R. China
}

Received April 17, 2019; Accepted October 14, 2019

DOI: $10.3892 / o r .2019 .7423$

\begin{abstract}
Lung cancer is a leading cause of cancer-related morbidity and mortality worldwide. Although there are currently various therapeutic strategies including surgery, chemotherapy and radiotherapy, lung cancer still results in high mortality with a 5-year survival rate of less than $20 \%$. The increasing need for new therapeutic targets and diagnostic/prognostic tools for lung cancer has promoted the demand for a better molecular and mechanistic understanding of its pathobiology. microRNA-30a-3p (miR-30a-3p) was recently recognized to be closely involved in the regulation of cancer cell invasion, migration and proliferation. However, the mechanistic role of miR-30a-3p in regulating the biological behavior of lung cancer, especially lung adenocarcinoma (LADC), is unknown. In the present study, we aimed to confirm the downregulation of miR-30a-3p in LADC tissues, and validate its functional impact on the pathogenesis of LADC via its molecular target, canopy fibroblast growth factor signaling regulator 2 (CNPY2), a known oncogene. Our data confirmed that CNPY2 was upregulated in LADC tissues, and the expression level of CNPY2 was correlated with the clinical outcomes of lung cancer patients. miR-30a-3p was confirmed as a key negative regulator of CNPY2 and reduced miR-30a-3p expression resulted in CNPY2 upregulation in LADC tissues. We then validated the functional outcome of miR-30a-3p in cancer pathobiology by the overexpression of miR-30a-3p in the LADC EKVX cell line. miR-30a-3p overexpression inhibited cancer cell proliferation, invasion and migration, by suppressing CNPY2 expression. In addition, miR-30a-3p inhibited epithelial-mesenchymal transition, a key feature of
\end{abstract}

Correspondence to: Professor Ting Wang, School of Laboratory Medicine, Department of Immunology, Bengbu Medical College, 2600 Dong Hai Avenue, Longzihu (University City), Bengbu, Anhui 233030, P.R. China

E-mail: 2014069@bbmc.edu.cn

Key words: microRNA, canopy fibroblast growth factor signaling regulator 2, lung adenocarcinoma, proliferation
LADC, via CNPY2 suppression. Taken together, these findings suggest that miR-30a-3p exerts a novel inhibitory role in the pathogenesis of LADC via CNPY2 downregulation, and the miR-30a-3p/CNPY2 pathway is a potential therapeutic target for human LADC.

\section{Introduction}

Lung cancer is a leading cause of cancer-related morbidity worldwide (1). As the major histological subtype of lung cancer, non-small cell lung cancer (NSCLC) has a prevalence of up to $80 \%$ in lung cancer patients. Of these patients, $50 \%$ are diagnosed with lung adenocarcinoma (LADC) (2). In clinical settings, the treatment of lung cancer mainly consists of surgery, chemotherapy and radiotherapy. However, the treatment efficacy of these conventional therapies varies and is unsatisfactory. Importantly, a group of drug candidates for molecular-targeted therapy have been tested in trials with promising beneficial effects (3-5). The increased need for new diagnostic, prognostic and therapeutic tools for NSCLC requires additional molecular and mechanistic studies regarding the pathobiology of NSCLC.

Canopy fibroblast growth factor signaling regulator 2 (CNPY2), a member of the CNPY protein family, is an endoplasmic reticulum (ER) luminal protein encoded by the CNPY2 gene (6). CNYP2 contributes to angiogenesis and the prevention of hypertrophic cardiomyopathy $(7,8)$. In addition, it modulates DNA replication, cellular invasion, blood vessel development, and metastasis of several malignancies including human prostate cancer, colorectal cancer, and renal cell carcinoma (9-12). In a recent study, an elevated CNPY2 expression level in NSCLC patients was correlated with poor survival (13). However, the biological function and regulation of CNPY2 in the pathogenesis of LADC are still unknown.

miR-30a, a member of the human miRNA family, is consistently downregulated in many malignancies including breast cancer, renal cell carcinoma, hepatocellular carcinoma, colorectal cancer and cervical cancer (14-18). Interestingly, it was controversially considered to be tumor-suppressive or oncogenic in distinct types of malignancies (19-21). We believe that the differential roles of miR-30 family members as tumor-suppressor genes or oncogenes, are dependent on the 
target gene in the particular type of cancer. There is a need to define the selective role of miR-30 family members in cancer regulation, in order to design more specific and potent therapies for cancer subtypes. Here, we focus on one member of the miR-30 family, miR-30a-3p, which is abundantly expressed with an unclear role in LADC, and has been identified as a CNPY2 regulator.

In the present study, for the first time, we investigated both CNPY2 and miR-30a-3p expression in LADC tissue samples and NSCLC cell lines. In addition, the molecular function and interaction of CNPY2 and miR-30a-3p in LADC cell lines were characterized. We identified $C N P Y 2$ as a target gene of miR-30a-3p with a tumor-suppressive function. Thus, miR-30a-3p activation or CNPY2 inhibition may be applicable for treating LADC.

\section{Materials and methods}

Patients and tissue samples. Tissue samples were collected from 64 LADC patients (mean age, 58 years; age range, 45-72 years; sex, 29 male and 35 female) undergoing surgery at the Department of Surgery, The First Affiliated Hospital, Bengbu Medical College between January 2012 and December 2017. The diagnosis of LADC was based on pathological findings. The samples were embedded in paraffin, and matched healthy lung tissues (adjacent tumors) served as controls. Patients with other types of tumors or chronic/acute lung diseases were excluded from this study. Each patient signed an informed consent. Sample handling was in line with the guidelines set out in the Declaration of Helsinki. The study protocols were in line with the guidelines of the Ethics Committee of Bengbu Medical College (Anhui, China).

TCGA analysis. To analyze the clinicopathologic parameters and overall survival curves of the LADC patients with high or low CNPY2 mRNA expression, data from The Cancer Genome Atlas (TCGA) dataset were retrieved and analyzed.

Cell culture and transfection. A LADC-derived cell line (EKVX cells) was provided by the Cell Bank of the Chinese Academy of Sciences (Shanghai, China). The control cells were normal human bronchial epithelial (HBE) cells purchased from Gaining Biotech (Shanghai, China). Cells were cultured in DMEM medium (Hyclone; GE Healthcare Life Sciences) containing $10 \%$ fetal bovine serum (FBS; Sigma-Aldrich; Merck $\mathrm{KGaA}$ ) at $37^{\circ} \mathrm{C}$ in $5 \% \mathrm{CO}_{2}$.

miR-30a-3p mimics and inhibitors were provided by GeneCopoeia (Guangzhou, China), together with the corresponding negative control vectors. The small interfering (si) RNA of CNPY2 was obtained from EnoGene (Nanjing, China). To induce overexpression and silencing of miR-30a-3p and CNPY2, EKVX cells were transfected with the corresponding vectors using a commercial Lipofectamine $2000 \mathrm{kit}$, according to standard protocols (Invitrogen; Thermo Fisher Scientific, Inc.).

Immunohistochemical staining evaluation. Following sample fixation using $10 \%$ formalin and embedding in paraffin, the sections $(3-\mu \mathrm{m})$ were subjected to immunohistochemical staining using an established protocol (22). In brief, the anti-human CNPY2 antibody (dilution 1:500; cat. no. ab233136; Abcam) was firstly used to stain the sections at $4^{\circ} \mathrm{C}$ overnight. Secondary antibodies were then added to the mixture. For visualization of the immunoreactions, a Vectastain ${ }^{\circledR}$ Elite avidin-biotin-peroxidase (ABC) complex (Vector Laboratories) kit was used. Non-specific binding was controlled by application of secondary-only negative controls.

A visual grading system was used for quantification of CNPY2 expression according to the extent and intensity of staining. The immunoreactivity score was determined as described previously (23). For immunoreactivity scoring of each sample, 10 visual fields were randomly selected from different areas. The average immunoreactivity score was obtained based on these 10 visual fields. High expression was defined as an immunoreactivity score of 4 or more. The slides were independently viewed by two experienced physicians blinded to clinicopathological information and outcome of the patients. In the cases of discrepancies, the data were reviewed simultaneously until a consensus was reached.

Real-time PCR. Trizol reagent was used for total RNA extraction from tissue samples and cultured cells. Synthesis of cDNA was conducted using the First-Strand cDNA synthesis kit (Thermo Fisher Scientific, Inc.). The miRNeasy Mini kit (Qiagen) was utilized to extract miRNA from the tissue samples and cell lines. cDNA was synthesized using the miScript II RT Kit (Qiagen). Quantitative real-time PCR was conducted on an Applied Biosystems ABI StepOnePlus Systems (Thermo Fisher Scientific, Inc.) using SYBR Green (Applied Biosystems; Thermo Fisher Scientific, Inc.). The internal controls for CNPY2 and miR-30a-3p were GAPDH and U6 snRNA. The quantification of miR-30a-3p and CNPY2 was performed based on the $2^{-\Delta \Delta \mathrm{Cq}}$ method (24). The specific primers for CNPY2, GAPDH, miR-30a-3p and U6 snRNA were as follows: CNPY2, 5'-ATCCTTCCACCCATCGCA AG-3' and 5'-AACATTGTCAGCCTCTCGGG-3'; GAPDH, 5'-GCACCGTCAAGGCTGAGAAC-3' and 5'-TGGTGAAGA CGCCAGTGGA-3'; miR-30a-3p, 5'-CGCTTTCAGTCGGAT GTTTG-3' and 5'-GTGCAGGGTCCGAGGT-3'; U6 snRNA, 5'-GCGCGTCGTGAAGCGTTC-3' and 5'-GTGCAGGGT CCGAGGT-3'.

Western blot analysis. Protein was extracted from the tissue samples and cells according to a previous report (21), followed by measurement with the BCA Protein Assay kit. The protein samples then underwent SDS-PAGE analysis, followed by transfer to polyvinylidene difluoride (PVDF) membranes. Subsequently, 5\% BSA was used to block the PVDF membranes at room temperature for $1 \mathrm{~h}$. The membranes were incubated with antibodies against CNPY2 (dilution 1:1,000; cat. no. ab233136; Abcam), N-cadherin (dilution 1:100; cat. no. ab18203; Abcam) or E-cadherin (dilution 1:100; cat. no. ab15148; Abcam) for $2 \mathrm{~h}$, and then incubated with HRP-conjugated secondary antibodies (HRP-goat anti-rabbit IgG, dilution 1:2,000; cat. no. ab205718; Abcam) for $1 \mathrm{~h}$. Finally, the enhanced chemiluminescence method was used to measure immunoreactivity using the ECL kit (Thermo Fisher Scientific, Inc.). The same membrane probed with 
$\beta$-actin (dilution 1:2,000; cat. no. ab8227; Abcam) served as the loading control.

Luciferase reporter assay. Wild-type (WT) and mutant (Mut) CNPY2 3'-untranslated region (UTR) were amplified and cloned into the pGL3-Basic vector purchased from Promega. Human 293T cells, purchased from the Cell Bank at the Chinese Academy of Sciences (Shanghai, China), were transfected with pGL3-Mut-3'-UTR-CNPY2 or pGL3-WT-3'-UTR-CNPY2, as well as miR-30a-3p mimic, miR-30a-3p inhibitor, control, or negative control vector. The pRL-TK vector (Promega) was used as an internal control for normalization of transfection efficacy. Approximately $48 \mathrm{~h}$ after transfection, the relative luciferase activity was evaluated using the dual-luciferase reporter assay system (Promega).

Invasion and migration assays. Quantitative assays of cellular migration and invasion were carried out in a commercial chamber (Corning) equipped with a polycarbonate filter $(8.0 \mu \mathrm{m})$ inserted into 24 -well plates. A Transwell system purchased from Corning was utilized to determine the invasion of EKVX cells. Matrigel (12.5 mg; BD Biosciences) in $50 \mathrm{ml}$ PBS was added to the filter. Cells suspended in serum-free medium $(100 \mu \mathrm{l})$ were transferred to the upper chamber. Subsequently, the lower chamber was filled with medium $(500 \mu \mathrm{l})$ supplemented with $10 \%$ FBS. After incubation at $37^{\circ} \mathrm{C}$ for $48 \mathrm{~h}$, the cells were removed from the upper surface of the filter using a cotton swab, and then penetrated to the lower surface of the filter followed by staining with crystal violet $(0.1 \%, 15 \mathrm{~min}$ at room temperature). The cells left on the lower side of each membrane were counted under a light microscope for five fields of view (magnification, $\mathrm{x} 200$ ).

Determination of cellular proliferation. Cell proliferation was measured with a commercial CCK-8 kit (Beyotime Institute of Biotechnology). The harvested cells $\left(1.0 \times 10^{4} /\right.$ well) were then seeded into 96 -well plates. Subsequently, $10 \mu 1$ of CCK-8 reagent was added to each well at $0,24,48$ and $72 \mathrm{~h}$, respectively. The mixture was then incubated for another $2 \mathrm{~h}$ at $37^{\circ} \mathrm{C}$, followed by determination of the absorbance at $450 \mathrm{~nm}$ using a microplate reader.

Colony formation assay. Approximately $48 \mathrm{~h}$ after cellular transfection, the cells were suspended in culture medium supplemented with $0.3 \%$ agarose. The cells were then plated on a layer containing $0.7 \%$ agarose in growth medium in a 6 -well plate and cultured for 14 days. The cells were then fixed using methanol (20 min) and stained with crystal violet dye $(0.1 \%$, $15 \mathrm{~min})$. Colonies consisting of more than 50 cells were counted as a single colony under a light inverted microscope (TS100; Nikon Corp.) and each assay was performed in triplicate.

Statistical analysis. All experiments were repeated at least three times, independently. Data are displayed as mean \pm standard error of the mean (SEM). SPSS 19.0 software (IBM Corp.) was used for data analysis. We evaluated the statistical significance with the performance of the one-way analysis of variance (ANOVA) test followed by Dunnett's post hoc test for the comparison of $>2$ groups. A P-value of $<0.05$ was considered statistically significant.

\section{Results}

CNPY2 is upregulated in LADC tissues and the expression level of CNPY2 correlates with clinical outcomes in LADC patients. CNPY2 expression in LADC tissues was first investigated. The protein expression level of CNPY2 in LADC samples was significantly increased compared to that of normal controls as shown by immunohistochemical analysis (Fig. 1A and B). To confirm and validate the relationship between clinicopathological parameters in LADC patients and CYPY2 expression, we analyzed data from The Cancer Genome Atlas (TCGA) database (https://cancergenome.nih. gov/). The clinicopathological and demographic features are documented in Table I. The TCGA cohort demonstrated consistent CNPY2 upregulation in LADC samples (Fig. 1C), regardless of clinical stage (Fig. 1D) or gender (Fig. 1E). The expression of CNPY2 was also correlated with LADC survival (Fig. 1F). These data confirmed that CNPY2 may serve as a marker of LADC, with some diagnostic and prognostic power in LADC, similar to that reported in other types of cancers $(10,11)$. However, it is not entirely clear how CNPY2 expression is modulated in LADC.

Downregulated miR-30a-3p contributes to CNPY2 dysregulation in $L A D C$. Following confirmation of CNPY2 upregulation in human LADC, we next characterized the mechanism of CNPY2 upregulation using molecular and cellular biology tools. The expression of miR-30a-3p was markedly reduced in LADC cells (EKVX) compared with that noted in the control non-cancer (HBE) cells (Fig. 2A), similar to previous findings in other types of cancers $(10,11)$. TargetScan (http://www.targetscan.org/) and miRanda bioinformatics databases (http://microrna.sanger.ac.uk/) identified the binding sequence for miR-30a-3p in the 3'-UTR of the CNPY2 gene (Fig. 2B). This binding and consequent expression inhibition was confirmed by a CNPY2 3'-UTR luciferase assay. The reporter luciferase activity with the wild-type (WT) CNPY2 3'-UTR vector was significantly reduced after treatment with miR-30a-3p mimics (Fig. 2C). However, the promoter luciferase activity with the mutant (Mut) CNPY2 3'-UTR (Fig. 2B) showed no significant changes (Fig. 2C) following treatment with miR-30a-3p mimics. EKVX cells were transfected with miR-30a-3p mimic or miR-30a-3p inhibitor to alter the expression of miR-30a-3p, and the transfection efficiency was identified by quantitative PCR analysis. The expression of miR-30a-3p was significantly augmented in the miR-30a-3p mimic-transfected cells but downregulated in miR-30a-3p inhibitor-transfected cells relative to the NC miRNA-transfected cells (Fig. S1). Then, the downregulation of CNPY2 mRNA by miR-30a-3p mimics and significant upregulation of CNPY2 mRNA mediated by miR-30a-3p inhibitors (Fig. 2D) were further confirmed by quantitative PCR. In addition, the regulation of CNPY2 expression at the protein level was validated by western blot analysis (Fig. 2E). These data directly demonstrate that miR-30a-3p inhibited CNPY2 expression, via specific miRNA binding at the post-transcriptional level, in LADC cells. We next confirmed the inverse expression pattern of miR-30a-3p and CNPY2 in LADC tissues. Compared to adjacent normal tissues, LADC tissues exhibited a significantly lower level of miR-30a-3p (Fig. 2F), and a higher level 

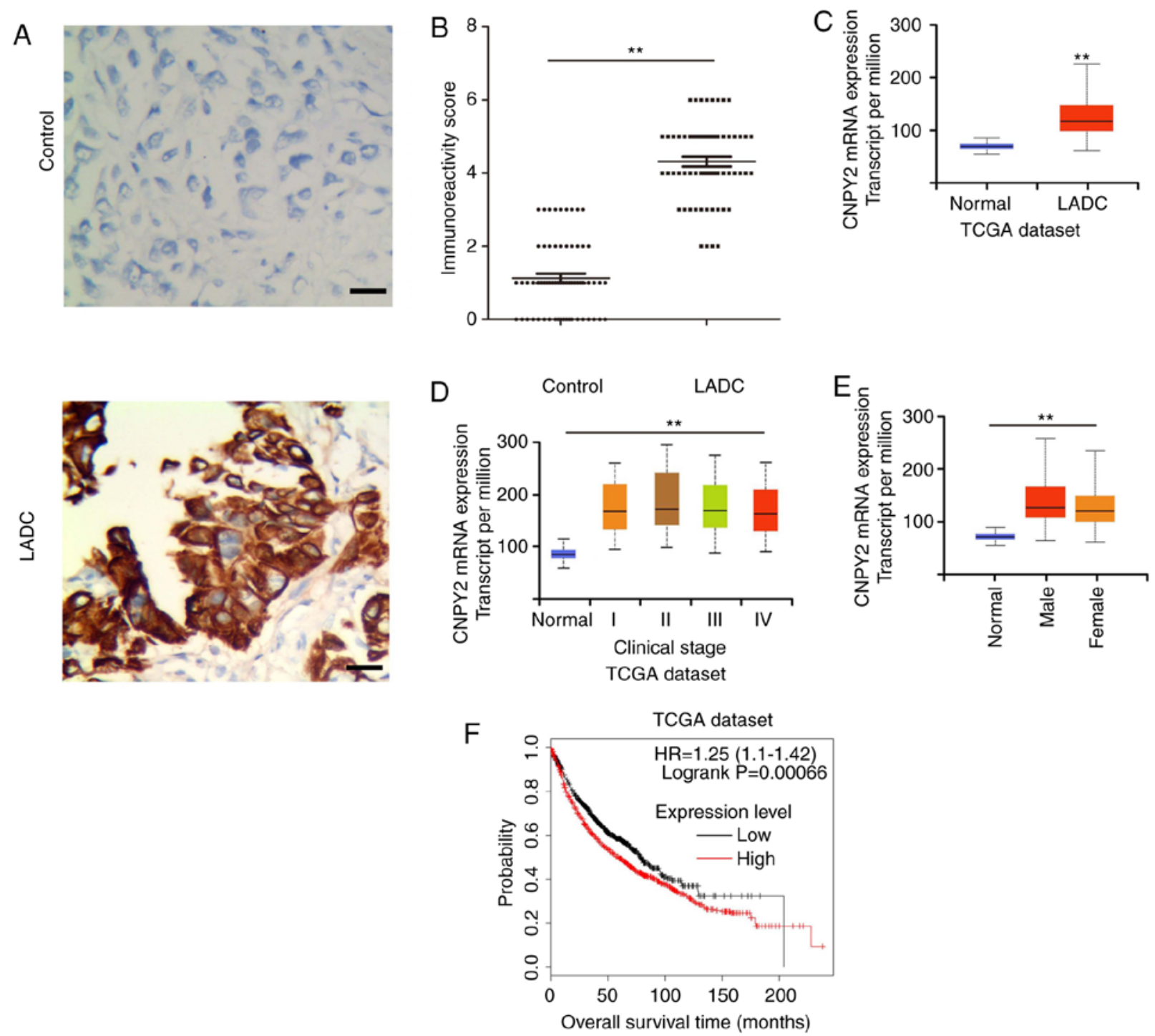

Figure 1.CNPY2 is upregulated in LADC tissues and the expression level of CNPY2 correlates with clinical outcome in LADC patients. (A) Immunohistochemical analysis of CNPY2 expression in LADC tissues ( $\mathrm{n}=64$, bottom panel) and matched normal lung tissues ( $\mathrm{n}=64$, top panel) (scale bar, $100 \mu \mathrm{m}$, magnification x200). (B) CNPY2 immunoreactivity scores were determined by blinded analysis. ${ }^{* *} \mathrm{P}<0.01$ vs. the control group. (C) Compared with normal control tissues $(\mathrm{n}=59)$, (the controls were obtained from paracancerous tissues in patients with LADC), CNPY2 was highly expressed in 515 LADC samples (TCGA cohort). (D) CNPY2 expression levels in patients with different clinical stages of LADC (TCGA cohort): Normal $(n=59)$, stage I $(n=277)$, II $(n=125)$, III ( $n=85)$ and IV $(n=28)$. (E) Expression of CNPY2 in LADC based on patient's sex (TCGA cohort): Normal $(n=59)$, male $(n=238)$ and female $(n=276)$. (F) The overall survival curve for patients with low vs. high CNPY2 using the publicly available Kaplan-Meier plotter database (www.kmplot.com): Low expression level (n=964), high expression level ( $\mathrm{n}=962)$. LADC, lung adenocarcinoma; CNPY2, canopy fibroblast growth factor signaling regulator 2; TCGA, The Cancer Genome Atlas.

of CNPY2 (Fig. 2G). A significant negative correlation was noted between miR-30a-3p and CNPY2 mRNA expression in tumor tissues following Spearman's rank correlation analysis $(\mathrm{r}=-0.8875, \mathrm{P}<0.05$, Fig. $2 \mathrm{H})$. Taken together, these findings show that miR-30a-3p is a direct negative regulator of CNPY2 in LADC under in vitro and in vivo conditions.

miR-30a-3p inhibits cellular proliferation, invasion and migration by targeting CNPY2. Following confirmation of the negative regulation of CNPY2 by miR-30a-3p in LADC both in vitro and in vivo, we next evaluated the functional outcome of reduced miR-30a-3p in LADC via CNPY2. miR-30a-3p overexpression (by miR-30a-3p mimics) significantly reduced cell proliferation (Fig. 3A), invasion (Fig. 3B), migration (Fig. 3C), and clone formation (Fig. 3D), whereas, miR-30a-3p inhibitors significantly increased cell proliferation (Fig. 3A), invasion
(Fig. 3B), migration (Fig. 3C), and clone formation (Fig. 3D). As CNPY2 is a direct target of miR-30a-3p in LADC, we then determined where CNPY2 is responsible for the oncogenic biological outcomes of miR-30a-3p. On this basis, rescue experiments were performed by co-transfecting miR-30a-3p inhibitor (to mimic the downregulation of miR-30a-3p in LADC cells) and selective CNPY2 silencing (si-CNPY2) in EKVX cells. In this study, the effectiveness of si-CNPY2 was confirmed by clearly reduced CNPY2 protein expression post-transfection in EKVX cells (Fig. 3F). In miR-30a-3p inhibitor-treated cells, si-CNPY2 partially reversed the increased cell proliferation rate, cell migration and invasion ability (Fig. 3B-E). We also observed similar results in A549 cells, another NSCLC cell line, where miR-30a-3p was responsible for suppression of proliferation and colony formation via CNPY2 (Fig. S2). These findings indicate, for the first time, 
Table I. Correlation analysis between the clinical features and expression of miR-30a-3p in the LADC cases.

\begin{tabular}{|c|c|c|c|c|c|}
\hline Variables & Total no. of pts $(\mathrm{N}=64)$ & miR-30a-3p low, $n$ & miR-30a-3p high, $n$ & $\chi^{2}$ & P-value \\
\hline Age (years) & & & & & 0.42 \\
\hline$\geq 50$ & 43 & 22 & 21 & 0.66 & \\
\hline$<50$ & 21 & 13 & 8 & & \\
\hline Sex & & & & & 0.66 \\
\hline Male & 29 & 15 & 14 & 0.19 & \\
\hline Female & 35 & 20 & 15 & & \\
\hline TNM stage & & & & & $0.0002^{\mathrm{b}}$ \\
\hline $\mathrm{I}+\mathrm{II}$ & 24 & 6 & 18 & 13.66 & \\
\hline III+IV & 40 & 29 & 11 & & \\
\hline Metastasis & & & & & $0.0002^{\mathrm{b}}$ \\
\hline Yes & 42 & 30 & 12 & 13.81 & \\
\hline No & 22 & 5 & 17 & & \\
\hline
\end{tabular}

${ }^{\mathrm{a} C h i}$-square test. ${ }^{\mathrm{b}} \mathrm{P}<0.05$. LDAC, lung adenocarcinoma; pts, patients; TNM, tumor node metastasis.
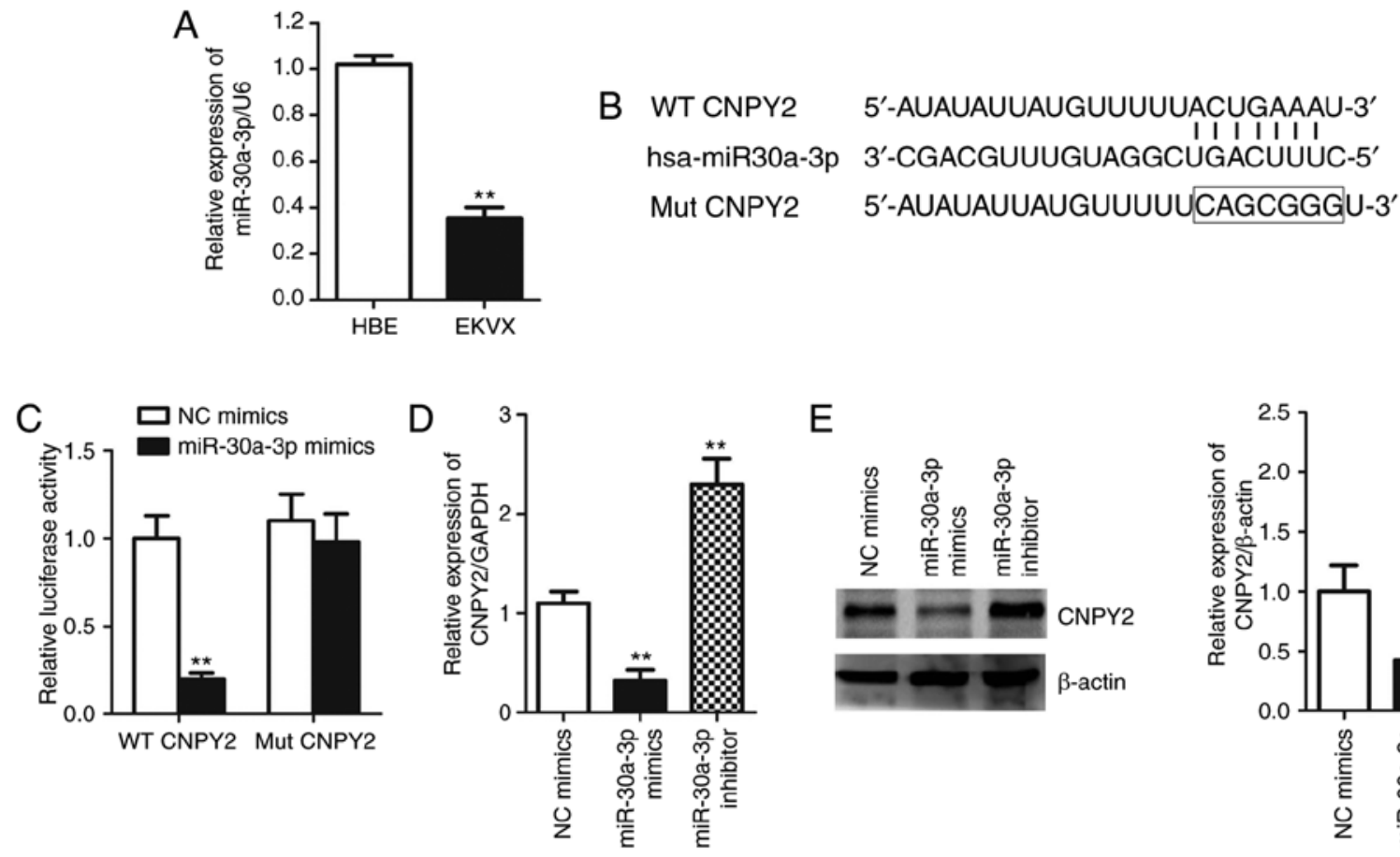

E
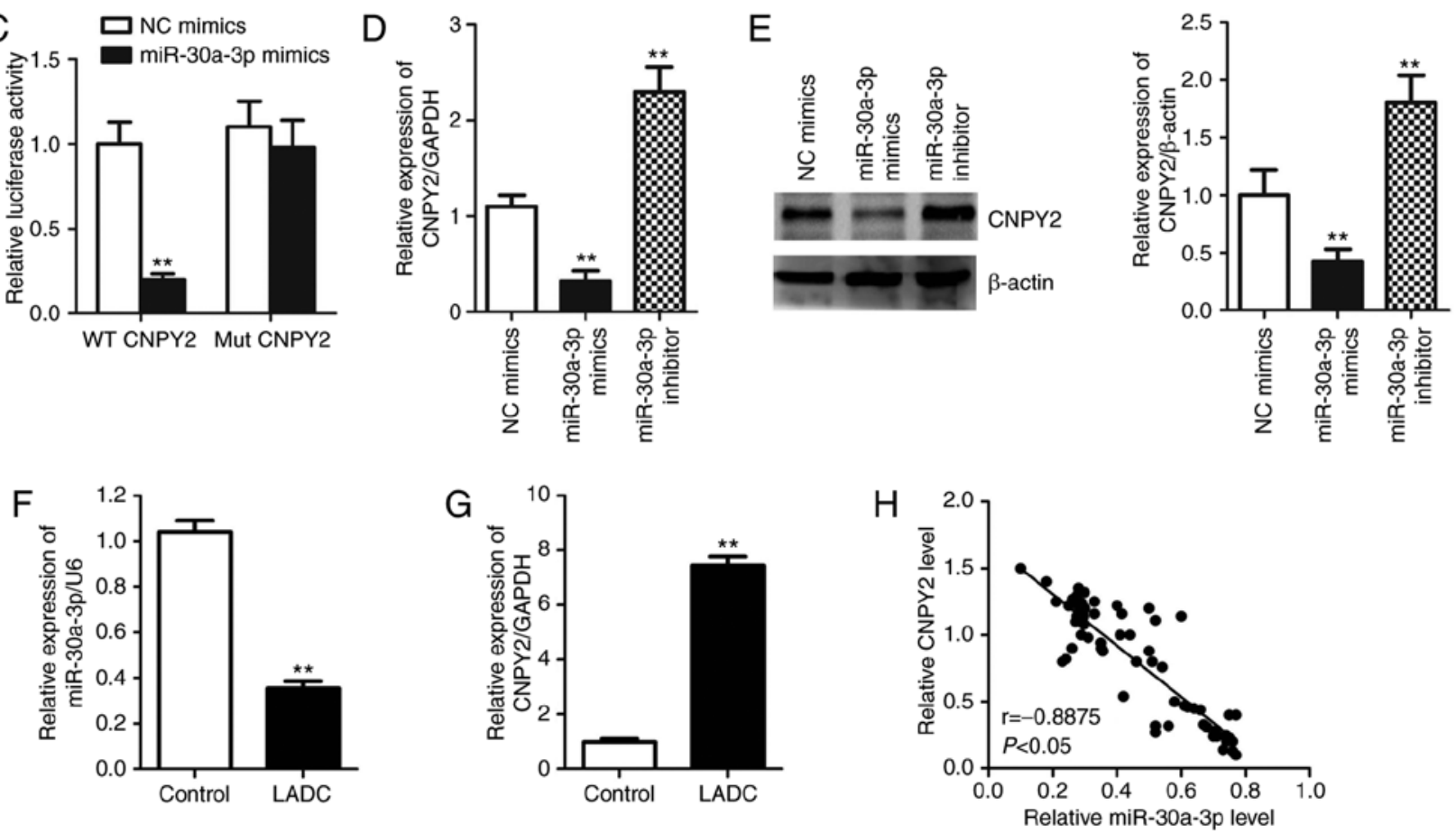

Figure 2. miR-30a-3p negatively regulates CNPY2 in LADC in vitro. (A) Expression of miR-30a-3p in the LADC cell line (EKVX) and the normal human bronchial epithelial (HBE) cell line were measured by RT-qPCR. CNPY2 is a direct target of miR-30a-3p. (B) Predicted miR-30a-3p binding sites in the 3'-UTR of wild-type (CNPY2-WT) and mutant (CNPY2-Mut) CNPY2 sequences. (C) Luciferase reporter assay using 293T cells co-transfected with CNPY2-WT (or CNPY2-Mut). Expression of CNPY2 in EVKX cells was measured by qPCR (D) or western blot analysis (E) post miR-30a-3p mimic, miR-30a-3p inhibitor, and control transfection. ${ }^{* *} \mathrm{P}<0.01$ vs. the control group. (F) miR-30a-3p was found to be downregulated and (G) CNPY2 was upregulated in LADC tissues ( $\left.\mathrm{n}=64\right)$ compared with normal tissues $(n=64)$. Data are presented as the mean \pm SEM. $(H)$ A significant correlation between the levels of miR-30a-3p and CNPY2 in LADC tissues $(n=64)$ was demonstrated using Spearman's rank correlation analysis $(r=-0.8875, P<0.05)$. LADC, lung adenocarcinoma; CNPY2, canopy fibroblast growth factor signaling regulator 2 . 

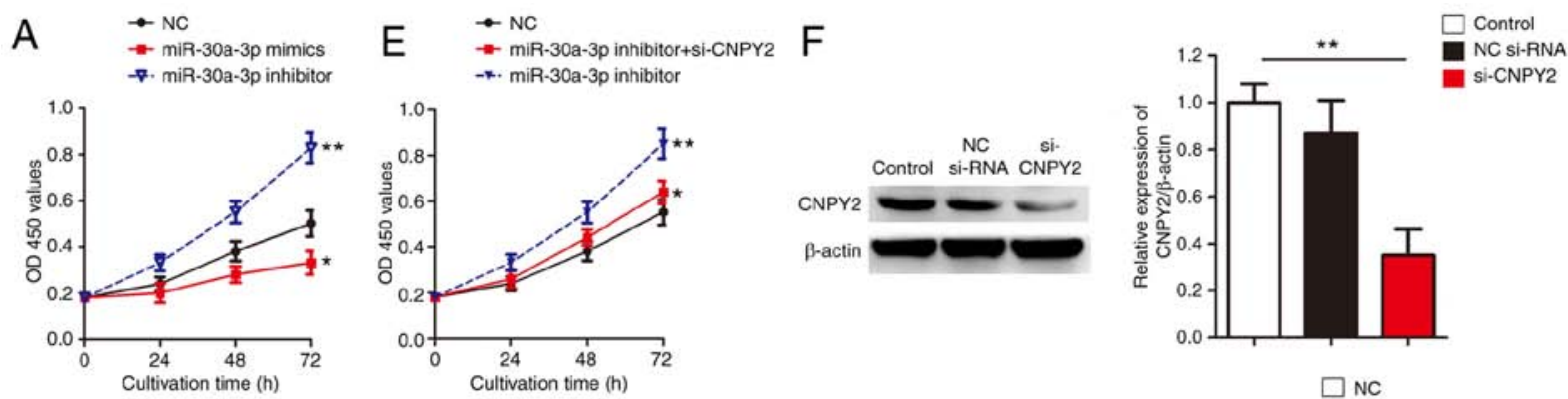

B

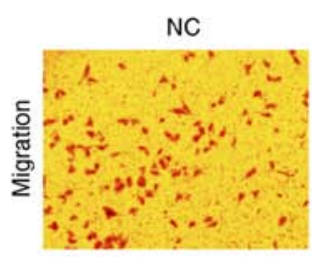

miR-30a-3p mimics
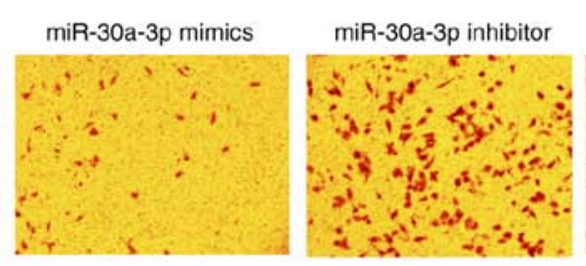

miR-30a-3p inhibitor si-CNPY2
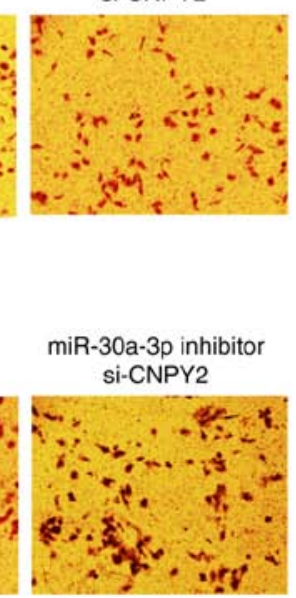

C

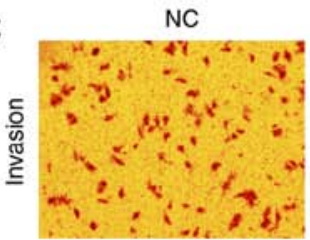

miR-30a-3p mimics
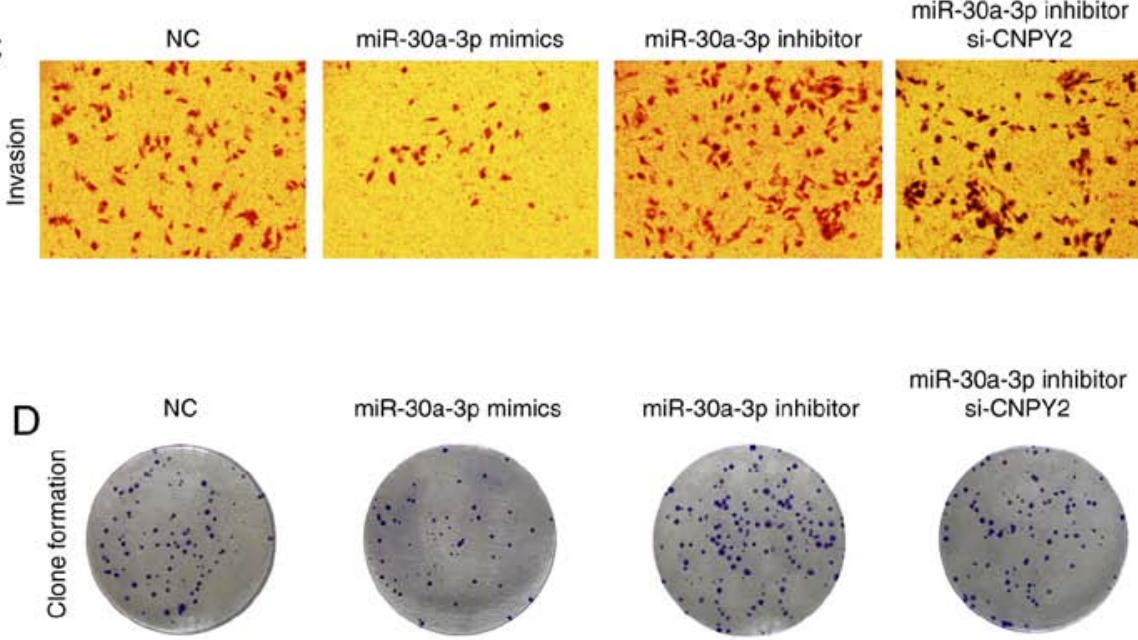

Figure 3. miR-30a-3p regulates LADC cell growth and invasion by targeting CNPY2. (A and E) Cell proliferation, (B) cell migration, (C) invasion, and (D) colony formation of EVKX cells were measured by the CCK-8 assay, Transwell assay and colony formation assay post si-CNPY2 and si-control (NC si-RNA) transfection $\left({ }^{* *} \mathrm{P}<0.01,{ }^{*} \mathrm{P}<0.05\right)$. (F) CNPY2 protein level in EVKX cells was assayed by western blot post si-CNPY2 or NC si-RNA transfection. LADC, lung adenocarcinoma; CNPY2, canopy fibroblast growth factor signaling regulator 2.

that miR-30a-3p inhibits LADC cell proliferation, invasion and migration by targeting CNPY2 in vitro.

miR-30a-3p targets CNPY2 in regulating EMT-associated proteins in vitro. CNPY2 was reported to enhance epithelial-mesenchymal transition (EMT), an essential step of metastasis in NSCLC (25). We next analyzed the roles of miR-30a-3p and CNPY2 in regulating EMT in EKVX cells. Consistent with a previous report (25), our data showed that CNPY2 knockdown led to downregulation of N-cadherin protein and upregulation of E-cadherin expression (Fig. 4A), suggesting EMT suppression. Notably, overexpression of miR-30a-3p exhibited effects similar to those for CNPY2 silencing, while miR-30a-3p inhibitor induced EMT, with increased N-cadherin and reduced E-cadherin (Fig. 4B). Our results further illustrate that CNPY2 serves as a target of miR-30a-3p in regulating EMT-associated proteins in LADC cells.

\section{Discussion}

microRNAs are a class of post-transcriptional regulatory molecules, some of which have been reported to be closely related to tumorigenesis by targeting a variety of tumor-associated genes (17-19,26). The miR-30a family, including miR-30a-3p and miR-30a-5p, is strongly related to the progression of different malignant tumors, including hepatocellular carcinoma, breast cancer $(14,15)$, colon cancer $(16)$, cervical cancer (17), and osteosarcoma (26). To note, miR-30a abnormally mediated tumor suppression or oncogenesis in various cancers $(17,19)$. However, only a few studies have investigated the roles of miR-30a-3p in lung adenocarcinoma (LADC). Our data demonstrated significant downregulation of miR-30a-3p in LADC tissues and EKVX cells. In addition, miR-30a-3p was found to play a suppressive role in LADC as its overexpression inhibited cellular proliferation, invasion and migration of 

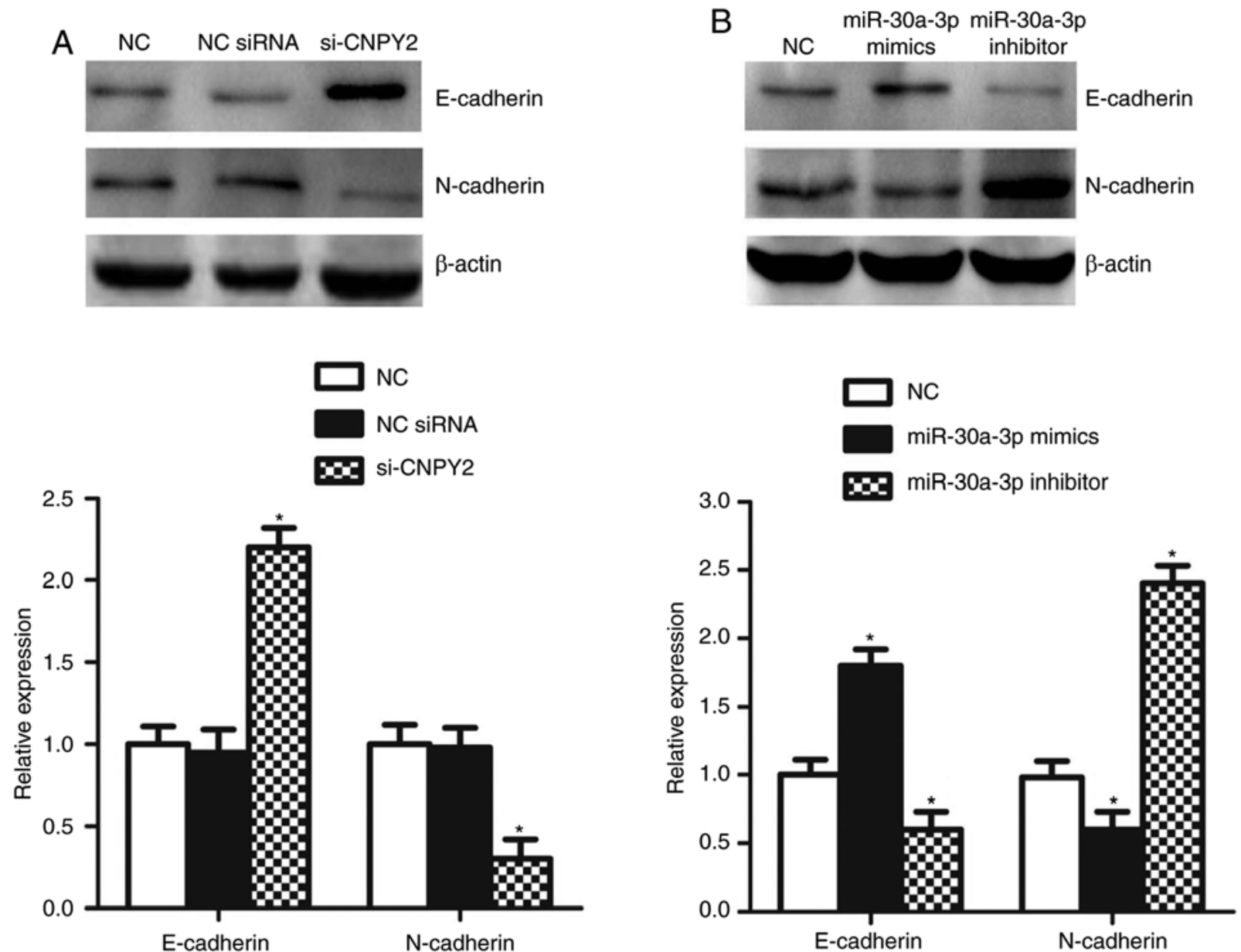

Figure 4. CNPY2 is a functional target of miR-30a-3p in regulating EMT. Knockdown of CNPY2 expression (A) or overexpression of miR-30a-3p (B) in EVKX cells, and EMT-related markers N-cadherin and E-cadherin were examined by western blotting in the different groups. " $\mathrm{P}<0.05$ compared with the control group. CNPY2, canopy fibroblast growth factor signaling regulator 2; EMT, epithelial-mesenchymal transition.

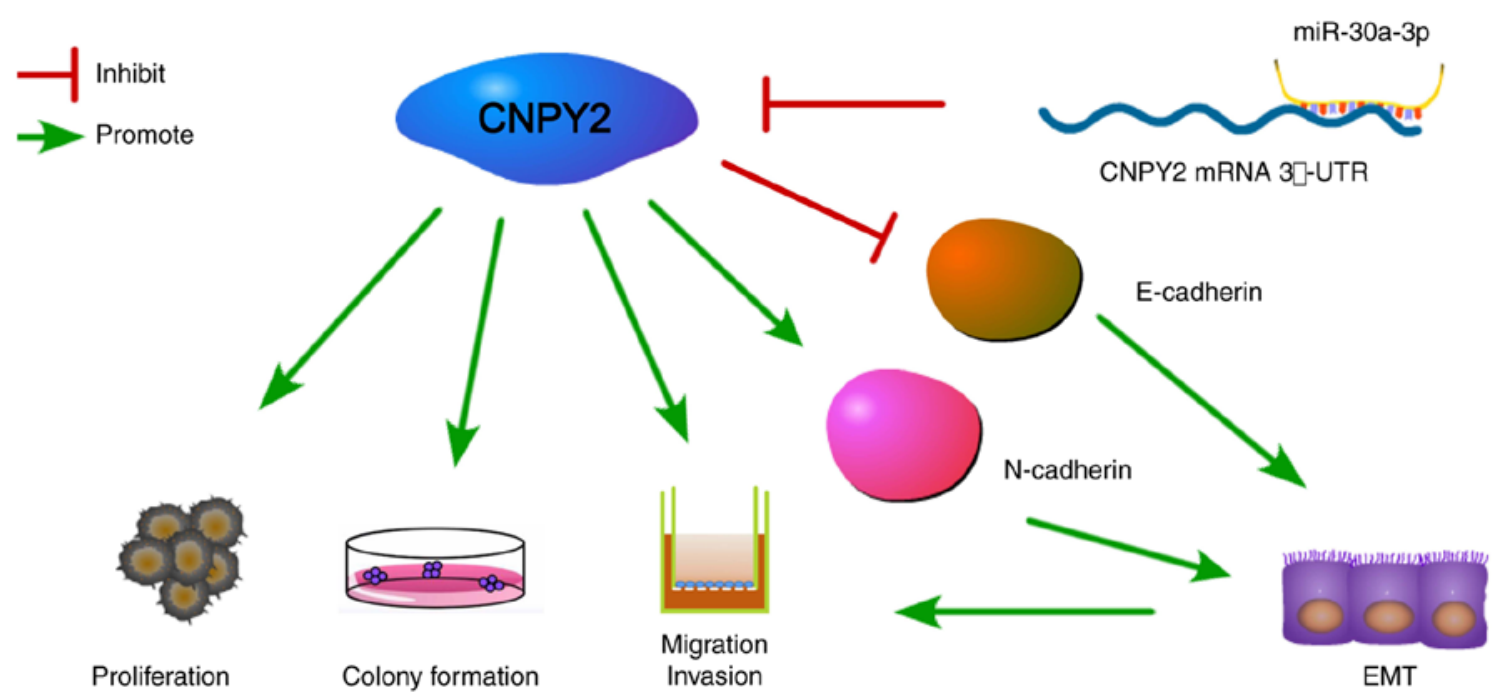

Figure 5. miR-30a-3p mediated the ability of CNPY2 to regulate the malignant behavior of LADC cells. LADC, lung adenocarcinoma; CNPY2, canopy fibroblast growth factor signaling regulator 2; EMT, epithelial-mesenchymal transition.

LADC cells. Importantly, we predicted and validated binding and negative regulation of the 3'UTR of canopy fibroblast growth factor signaling regulator 2 (CNPY2) by miR-30a-3p with various computational biology and molecular biology tools. We investigated the relationship between CYPY2 and the clinicopathological parameters of LADC by analyzing the TCGA database, which indicated that high expression of CNPY2 in LADC samples was significantly correlated with 
staging and metastasis. In recent studies, CNPY2 was found to promote tumor growth and angiogenesis by activating p53 in human colorectal cancer and renal cell carcinoma $(10,11)$. For example, CNPY2, overexpressed in non-small cell lung cancer (NSCLC) tissues, was negatively correlated with the prognosis of NSCLC patients, while its overexpression inhibited cisplatin-induced apoptosis of a NSCLC cell line (13). We then investigated CNPY2 expression in EKVX cells following artificial alteration of miR-30a-3p levels. Western blotting showed that miR-30a-3p was involved in the negative regulation of CNPY2. Furthermore, miR-30a-3p regulated cellular viability and invasion of LADC cells, which indicated that miR-30a-3p may be associated with the pathogenesis of LADC by modulating the expression of CNPY2.

NSCLC mainly includes squamous cell carcinoma and adenocarcinoma, and is considered one of the major causes of cancer-related death. Metastasis is involved and responsible for the high mortality rate of LADC patients. Therefore, it is necessary to understand the mechanism of invasion and metastasis of LADC, which may be beneficial for molecular therapy with the aim of inhibiting cancer progression and metastasis. Several mechanisms have been reported to be related to the local progression and metastasis of NSCLC, particularly EMT $(27,28)$. In this study, overexpression of miR-30a-3p or knockdown of CNPY2 in EKVX cells resulted in downregulation of $\mathrm{N}$-cadherin and upregulation of E-cadherin (Fig. 5). Therefore, we confirmed that in EKVX cells, CNPY2 is a target of miR-30a-3p in the regulation of EMT-associated proteins. Our results are consistent with those of Dou et al, who found that CNPY2 contributes to EMT through activation of the AKT/GSK3 $\beta$ pathway in NSCLC (25). The changes in protein expression led to a decrease in cell-cell adhesion, resulting in cancer cell proliferation and EMT. Therefore, miR-30a-3p activation or CNPY2 inhibition may be a novel strategy for the treatment of LADC.

The present study provides clear evidence that i) $\mathrm{miR}-30 \mathrm{a}-3 \mathrm{p}$ is reduced in LADC, ii) suppression of miR-30a-3p leads to upregulation of CNPY2, a known oncogene, iii) miR-30a-3p modulation regulates cancer-related cellular function in LADC cells, and iv) the biological functions of miR-30a-3p, at least in part, are dependent on CNPY2. However, due to the nonselective nature of the epigenetic regulatory role of miRNA, the provided data do not distinguish the effects of miR-30a-3p on CNPY2 from those of other targets. miR-30a-3p is reported to bind and downregulate the expression of WNT2, a central regulator of the WNT pathway (29). In order to better define the mechanistic role of miR-30a-3p in different cancers, a more selective approach is required, such as 'Target Protectors' (30), to define the selective role of an miRNA-targeted mRNA in a specific phenotypic readout. This will be a future research direction based on the results obtained in the current study.

The human miR30a gene encodes two miRNA types including miR-30a-3p and miR-30a-5p (GENECARDS: https://www.genecards.org/), with different sequences and targets. miR-30a-5p has been extensively studied, with more than twice the number of peer-reviewed research articles published up to 2019 compared to miR-30a-3p. In NSCLC, miR-30a-5p is known as a potential biomarker (31) with significant downregulation, similar to miR-30a-3p. This is the first study on the association between miR-30a-3p and CNPY2 in LADC. We confirmed that CNPY2 mRNA selectively binds to miR-30a-3p, compared to miR-30a-5p, further confirming the selective regulation of CNPY2 via miR-30a-3p in LADC. A consistent downregulation of miR-30a in cancer tissues has been observed (32), although the mechanism leading to this downregulation has not been defined. It has been suggested that dysregulated MIR $30 \mathrm{~A}$ gene promoter methylation is involved (33). Further studies are needed to define the mechanism of miR-30a-3p suppression and selectivity of the miRNA target in various types of cancer.

In conclusion, the present study demonstrated that miR-30a-3p acts as a tumor inhibitor in LADC via CNPY2 downregulation. In the future, potential molecular therapies targeting the miRNA/CNPY2 axis should be explored as novel strategies with which to treat LADC.

\section{Acknowledgements}

Not applicable.

\section{Funding}

The present study was supported by the National Natural Science Foundation of China (grant nos. 81772493 and 81570011), Science and Technology Program of Anhui Province (Key Laboratories project: 1606c08225, 2016080503B035, 2017070503B037) and Bengbu Medical College Science and Technology Development Fund (BYKY1644).

\section{Availability of data and materials}

The TCGA data which was used to support this study is available at TCGA website (https://cancergenome.nih.gov/).

\section{Authors' contributions}

HW and TW participated in the design and coordination of the study. DK, RL and XX performed the experiments. XX and RL performed the statistical analysis. HW and ZQ wrote the paper. XW, HW and TW reviewed and edited the manuscript. All authors read and approved the final manuscript.

\section{Ethics approval and consent to participate}

Sample handling was in line with the guidelines set out in the Declaration of Helsinki. The study protocols were in line with the guidelines of the Ethics Committee of Bengbu Medical College (Anhui, China).

\section{Patient consent for publication}

Not applicable.

\section{Competing interests}

The authors declare that they have no competing interests.

\section{References}

1. Bray F, Ferlay J, Soerjomataram I, Siegel RL, Torre LA and Jemal A: Global cancer statistics 2018: GLOBOCAN estimates of incidence and mortality worldwide for 36 cancers in 185 countries. CA Cancer J Clin 68: 394-424, 2018. 
2. Siegel RL, Miller KD and Jemal A: Cancer statistics, 2015. CA Cancer J Clin 65: 5-29, 2015.

3. Maemondo M, Inoue A, Kobayashi K, Sugawara S, Oizumi S, Isobe $\mathrm{H}$, Gemma A, Harada M, Yoshizawa H, Kinoshita I, et al: North-East japan study group: Gefitinib or chemotherapy for non-small-cell lung cancer with mutated EGFR. N Engl J Med 362: 2380-2388, 2010.

4. Fukuoka M, Wu YL, Thongprasert S, Sunpaweravong P, Leong SS, Sriuranpong V, Chao TY, Nakagawa K, Chu DT Saijo N, et al: Biomarker analyses and final overall survival results from a phase III, randomized, open-label, first-line study of gefitinib versus carboplatin/paclitaxel in clinically selected patients with advanced non-small-cell lung cancer in asia (IPASS). J Clin Oncol 29: 2866-2874, 2011.

5. Jänne PA, Yang JC, Kim DW, Planchard D, Ohe Y, Ramalingam SS, Ahn MJ, Kim SW, Su WC, Horn L, et al: AZD9291 in EGFR inhibitor-resistant non-small-cell lung cancer. N Engl J Med 372: 1689-1699, 2015.

6. Bruhn H: A short guided tour through functional and structural features of saposin-like proteins. Biochem J 389: 249-257, 2005

7. Guo J, Zhang Y, Mihic A, Li SH, Sun Z, Shao Z, Wu J, Weisel RD and Li RK: A secreted protein (Canopy 2, CNPY2) enhances angiogenesis and promotes smooth muscle cell migration and proliferation. Cardiovasc Res 105: 383-393, 2015.

8. Guo J, Mihic A, Wu J, Zhang Y, Singh K, Dhingra S, Weisel RD and Li RK: Canopy 2 attenuates the transition from compensatory hypertrophy to dilated heart failure in hypertrophic cardiomyopathy. Eur Heart J 36: 2530-2540, 2015.

9. Ito S, Ueda T, Ueno A, Nakagawa H, Taniguchi $\mathrm{H}$, Kayukawa $\mathrm{N}$ and Miki T: A genetic screen in drosophila for regulators of human prostate cancer progression. Biochem Biophys Res Commun 451: 548-555, 2014

10. Yan P, Gong H, Zhai X, Feng Y, Wu J, He S, Guo J, Wang X, Guo R, Xie J and Li RK: Decreasing CNPY2 expression diminishes colorectal tumor growth and development through activation of p53 pathway. Am J Pathol 186: 1015-1024, 2016.

11. Taniguchi H, Ito S, Ueda T, Morioka Y, Kayukawa N, Ueno A Nakagawa H, Fujihara A, Ushijima S, Kanazawa M, et al: CNPY2 promoted the proliferation of renal cell carcinoma cells and increased the expression of TP53. Biochem Biophys Res Commun 485: 267-271, 2017.

12. Shimura M, Mizuma M, Takadate T, Katoh Y, Suzuki T, Iseki M, Hata T, Aoki S, Suzuki Y, Sakata N, et al: A novel liver metastasis-correlated protein of pancreatic neuroendocrine neoplasm (PanNEN) discovered by proteomic analysis. Oncotarget 9: 24291-24303, 2018.

13. Yu D, Qin Y, Jun-Qiang L and Shun-Lin G: CNPY2 enhances resistance to apoptosis induced by cisplatin via activation of NF-kB pathway in human non-small cell lung cancer. Biomed Pharmacother 103: 1658-1663, 2018.

14. Fu J, Xu X, Kang L, Zhou L, Wang S, Lu J, Cheng L, Fan Z, Yuan B, Tian P, et al: MiR-30a suppresses breast cancer cell proliferation and migration by targeting eya2. Biochem Biophys Res Commun 445: 314-319, 2014.

15. Wang W, Lin H, Zhou L, Zhu Q, Gao S, Xie H, Liu Z, Xu Z, Wei J, Huang $X$ and Zheng S: MicroRNA-30a-3p inhibits tumor proliferation, invasiveness and metastasis and is downregulated in hepatocellular carcinoma. Eur J Surg Oncol 40: 1586-1594, 2014.

16. Liu M, Huang F, Zhang D, Ju J, Wu XB, Wang Y, Wang Y, Wu Y, Nie M, Li Z, et al: Heterochromatin protein HP1 $\gamma$ promotes colorectal cancer progression and is regulated by miR-30a. Cancer Res 75: 4593-4604, 2015.

17. Liu Z, Chen L, Zhang X, Xu X, Xing H, Zhang Y, Li W, Yu H, Zeng J and Jia J: RUNX3 regulates vimentin expression via miR-30a during epithelial-mesenchymal transition in gastric cancer cells. J Cell Mol Med 18: 610-623, 2014.
18. Zhao J, Li B, Shu C, Ma Y and Gong Y: Downregulation of miR-30a is associated with proliferation and invasion via targeting MEF2D in cervical cancer. Oncol Lett 14: 7437-7442, 2017.

19. Wang Z, Dai X, Chen Y, Sun C, Zhu Q, Zhao H, Liu G, Huang Q and Lan Q: MiR-30a-5p is induced by Wnt/3-catenin pathway and promotes glioma cell invasion by repressing NCAM. Biochem Biophys Res Commun 465: 374-380, 2015.

20. Wang HY, Li YY, Fu S, Wang XP, Huang MY, Zhang X, Shao Q, Deng L, Zeng MS, Zeng YX and Shao JY: MicroRNA-30a promotes invasiveness and metastasis in vitro and in vivo through epithelial-mesenchymal transition and results in poor survival of nasopharyngeal carcinoma patients. Exp Biol Med (Maywood) 239: 891-898, 2014.

21. Park D, Kim H, Kim Y and Jeoung D: MiR-30a regulates the expression of CAGE and p53 and regulates the response to anti-cancer drugs. Mol Cells 39: 299-309, 2016.

22. Feng Z, Gan H, Cai Z, Li N, Yang Z, Lu G and Chen J: Aberrant expression of hypoxia-inducible factor $1 \alpha$, TWIST and E-cadherin is associated with aggressive tumor phenotypes in endometrioid endometrial carcinoma. Jpn J Clin Oncol 43: 396-403, 2013.

23. Wang H, Liu H, Min S, Shen Y, Li W and Wang X: CDK16 overexpressed in non-small cell lung cancer and regulates cancer cell growth and apoptosis via a p27-dependent mechanism. Biomed Pharmacother 103: 399-405, 2018.

24. Livak KJ and Schmittgen TD: Analysis of relative gene expression data using real-time quantitative PCR and the 2(-Delta Delta C(T)) method. Methods 25: 402-408, 2001.

25. Dou Y, Lei JQ, Guo SL, Zhao D, Yue HM and Yu Q: The CNPY2 enhances epithelial-mesenchymal transition via activating the AKT/GSK3 $\beta$ pathway in non-small cell lung cancer. Cell Biol Int 42: 959-964, 2018.

26. Zhong B, Guo S, Zhang W, Zhang C, Wang Y and Zhang C: Bioinformatics prediction of miR-30a targets and its inhibition of cell proliferation of osteosarcoma by up-regulating the expression of PTEN. BMC Med Genomics 10: 64, 2017.

27. Chaffer CL, San Juan BP, Lim E and Weinberg RA: EMT, cell plasticity and metastasis. Cancer Metastasis Rev 35: 645-654, 2016.

28. Santamaria PG, Moreno-Bueno G, Portillo F and Cano A: EMT: Present and future in clinical oncology. Mol Oncol 11: 718-738, 2017.

29. Qi B, Wang Y, Chen ZJ, Li XN, Qi Y, Yang Y, Cui GH, Guo HZ, $\mathrm{Li}$ WH and Zhao S: Down-regulation of miR-30a-3p/5p promotes esophageal squamous cell carcinoma cell proliferation by activating the Wnt signaling pathway. World J Gastroenterol 23: 7965-7977, 2017.

30. Staton AA and Giraldez AJ: Use of target protector morpholinos to analyze the physiological roles of specific miRNA-mRNA pairs in vivo. Nat Protoc 6: 2035-2049, 2011.

31. Switlik W, Karbownik MS, Suwalski M, Kozak J and Szemraj J: MiR-30a-5p together with miR-210-3p as a promising biomarker for non-small cell lung cancer: A preliminary study. Cancer Biomark 21: 479-488, 2018.

32. Zhu Q, Li H, Li Y and Jiang L: MicroRNA-30a functions as tumor suppressor and inhibits the proliferation and invasion of prostate cancer cells by down-regulation of SIX1. Hum Cell 30: 290-299, 2017.

33. Han X, Zhen S, Ye Z, Lu J, Wang L, Li P, Li J, Zheng X, Li H, Chen W and Zhao L: A feedback loop between miR-30a/c-5p and DNMT1 mediates cisplatin resistance in ovarian cancer cells Cell Physiol Biochem 41: 973-986, 2017. 